\title{
BMJ Global Health Health aid projects have both expanded and constrained the capacity of health facilities to deliver malaria services to under-five children in Malawi
}

\author{
Carrie B Dolan
}

To cite: Dolan CB. Health aid projects have both expanded and constrained the capacity of health facilities to deliver malaria services to under-five children in Malawi. BMJ Glob Health 2018;3:e001051. doi:10.1136/ bmjgh-2018-001051

Handling editor Valery Ridde

- Additional material is published online only. To view please visit the journal online (http://dx.doi.org/10.1136/ bmjgh-2018-001051).

Received 18 July 2018 Revised 8 October 2018 Accepted 6 November 2018

Check for updates

(c) Author(s) (or their employer(s)) 2018. Re-use permitted under CC BY-NC. No commercial re-use. See rights and permissions. Published by BMJ.

Department of Kinesiology and Health Sciences, College of William and Mary, Williamsburg, Virginia, USA

Correspondence to

Dr Carrie B Dolan;

cbdolan@wm.edu

\section{ABSTRACT}

Objective This article examines the potential pathways health aid may use to influence the availability of malaria services at a facility level and the utilisation of malaria services for children under five in Malawi.

Methods This work is grounded in a health services research theoretical model and combines a subnational census of health services available at Malawi health facilities with individual-level data on health service utilisation and the Government of Malawi's official source of data about health aid allocation at a childlevel $(n=2171)$. Logistic and multinomial logistic models were used to assess the relationship between health aid, malaria service readiness and malaria service utilisation. Models were adjusted for predisposing, enabling and need factors and accounted for the complex relationship using a mediation approach.

Results The evidence presented suggests that health aid translates into increased diagnostic capacity, but not overall or training readiness. Results indicate that increasing aid projects in a region boost its facilities' diagnostic readiness, increasing each facility's relative likelihood of having a medium level of diagnostic readiness by $12 \%$ (relative risk $(\mathrm{RR})=1.118 ; 95 \% \mathrm{Cl} 1.060$ to 1.179 ) and its likelihood of having a high level of readiness by $23 \%$ ( $\mathrm{RR}=1.230 ; 95 \% \mathrm{Cl} 1.161$ to 1.303 ), but decreasing its readiness to provide training by $8 \%$ ( $R R=0.925 ; 95 \% \mathrm{Cl}$ 0.879 to 0.974 ).

Conclusion The results of this research highlight the fact that health aid is working to increase malaria diagnostic capacity at a facility level, but that increasing facility readiness to implement the diagnostic tests has been neglected.

\section{BACKGROUND}

Early diagnosis and prompt treatment of malaria saves lives, especially among children under five, who are particularly susceptible to the disease. ${ }^{1}$ Research shows that health aid for malaria programmes increases diagnosis and treatment at the national level, but the subnational level has been underexplored. ${ }^{2}$ This article addresses this gap by examining the potential pathways by which health aid

\section{Key questions}

What is already known?

- Accurate and early diagnosis and treatment are key strategies to control and prevent malaria among children under five. Yet, very little is known about the influence of health aid on the utilisation of these services in Malawi.

What are the new findings?

- This study is unique because it joins individual and facility level data at a subnational level with the purpose of examining the potential pathways by which health aid may influence utilisation of malaria services among children under five in Malawi.

- Evidence suggests that health aid does contribute to the availability of diagnostic tools, but not an overall ability to implement these tools.

What do the new findings imply?

- This study highlights a need to improve the availability of malaria skills training at a facility level. Funding mechanisms for malaria should provide consistent and comprehensive training in the use of malaria diagnostic tools so that healthcare workers can implement these resources consistently and reliably.

may influence utilisation of malaria services among children under five. It focuses on Malawi, which provides an excellent case for this investigation because of a high incidence of malaria and extensive dependence on donors, as much as $73.8 \%$ of the health sector relied on external resources in 2016 . $^{3}$ Donor reports claim that development efforts have improved health outcomes, but note some aid funds have been unaccounted for or poorly documented. Health spending as a percentage of the national budget has declined from $12 \%$ in $2013-2014$ to $9 \%$ in the 2016-2017 budget mostly due to the impact of the 2013 'Cashgate' spending scandal. ${ }^{34}$ Withdrawal of budget support by donors in 2013 has attenuated aid impacts, resulting in 
a lower availability of medicines and health personnel. Research examining the association between allocation of donor dollars for health and child health outcomes, in Malawi remains limited. One study examined donor-financed expanded coverage of malaria interventions in four hospitals in Malawi, only to find malaria admission rates increasing or remaining unchanged from 2000 to $2010 .^{5}$ This suggests a need to examine the results of funding for malaria services.

This article is the first to combine a subnational census of health services available at Malawi health facilities with individual-level data on health service utilisation and Malawi's official source of statistics and data about health aid allocation. Questions that investigate health aid and service utilisation in Malawi are useful because they take into account poor health conditions, significant donor attention and possible evidence of aid inefficiencies, such as corruption. The current study supports an existing need in the literature to use higher-resolution subnational data in order to understand the potential pathways by which health aid may influence utilisation of malaria services. ${ }^{5}$ The first of four research questions is, does the allocation of health aid correlate with utilisation of malaria services among children under 5 years old? Second, is health aid associated with health facility in readiness to provide malaria services? Third, does malaria facility readiness correlate with use of malaria services? Fourth, what is the effect of health aid on malaria service utilisation controlling for malaria service readiness? This research tests the hypothesis that health aid boosts a facility's readiness to provide malaria services, thereby increasing utilisation of malaria services by children under five residing in the facility's service area.

\section{METHODS}

\section{Conceptual model}

The Andersen and Newman Framework of Health Services Utilisation was adapted for this study (online supplementary appendix A). ${ }^{6}$ The framework treats healthcare use as a result of individual decisions driven by societal resources and service availability. The model states that societal determinants of utilisation affect individual determinants such as predisposition to use services, ability to use them and the need for them both directly and through the health services system. ${ }^{6}$ This paper adapts this behavioural model of health service utilisation to examine the relationship between both social and demographic factors and the individual utilisation of malaria services (online supplementary appendix A).

\section{Analysis}

The analyses used to test the relationships as described in the conceptual model above combine six sources of subnational, spatial-referenced data. Online supplementary appendix B describes each of the variables used in the empirical approach, which construct what the variables relate to in the conceptual model, as well as the data source.

To test the hypothesis that health aid boosts malaria service utilisation indirectly via increasing facilities' readiness to provide malaria services, this research uses logistic and multinomial logistic regression within a mediation approach. This allows examination of the paths by which aid can plausibly effect utilisation. To assess mediation, I use Baron and Kenney's four equations, depicted in figure 1. In this figure, the rectangles and the arrows represent the relationship between health aid (X), malaria service readiness $(\mathrm{M})$ and malaria service utilisation (Y) ${ }^{7}$ This figure also uses notation most widely applied in mediation analysis, with Pathway A representing the relationship between health aid and malaria service readiness (Research Question 2), Pathway B representing the effect of malaria service readiness on malaria service utilisation controlling for health aid (Research Question 3), Pathway $\mathrm{C}$ representing the relationship between health aid and malaria service utilisation (Research Question 1) and, finally, Pathway C' is the effect of health aid on malaria service utilisation controlling for malaria service readiness (Research Question 4). The conceptual model that guides the empirical approach also details and labels these relationships (online supplementary appendix A). Online supplementary appendix $\mathrm{C}$ provides the empirical approach to the mediation analysis, including equations.

\section{Outcomes}

The outcome variables for the logistic analyses are dichotomous, indicating whether or not a child under five who had a fever in the last 2 weeks received (1) advice or treatment, (2) finger or heel stick, (3) malaria artemisinin-based combination therapy (ACT) or (4) any malaria service from the 2012 Malawi Malaria Indicator Survey (MIS). The construction of these outcomes, as online supplementary appendix D describes, are based on guidance outlined in the Household Survey Indicators for Malaria Control, Section 3.3 Case Management among Children under 5 years old. ${ }^{8}$ The outcome for the multinomial logistic model is an index of malaria service readiness (low, medium, high) that is a quality measure designed to determine the capacity of health facilities to provide malaria services.

\section{Key independent variables}

The logistic models use four key independent variables. The first is health aid. The second is an index of facility readiness to provide malaria services. The last two are a subset of facility readiness measures, which include readiness to provide malaria diagnostic services and readiness to provide malaria training. In the multinomial logistic model, the key independent variable was health aid.

\section{Health aid}

The first key independent variable is health aid which includes 15 projects sited in 108 locations; totalling USD $\$ 120 \mathrm{M}$ in cumulative commitments and 

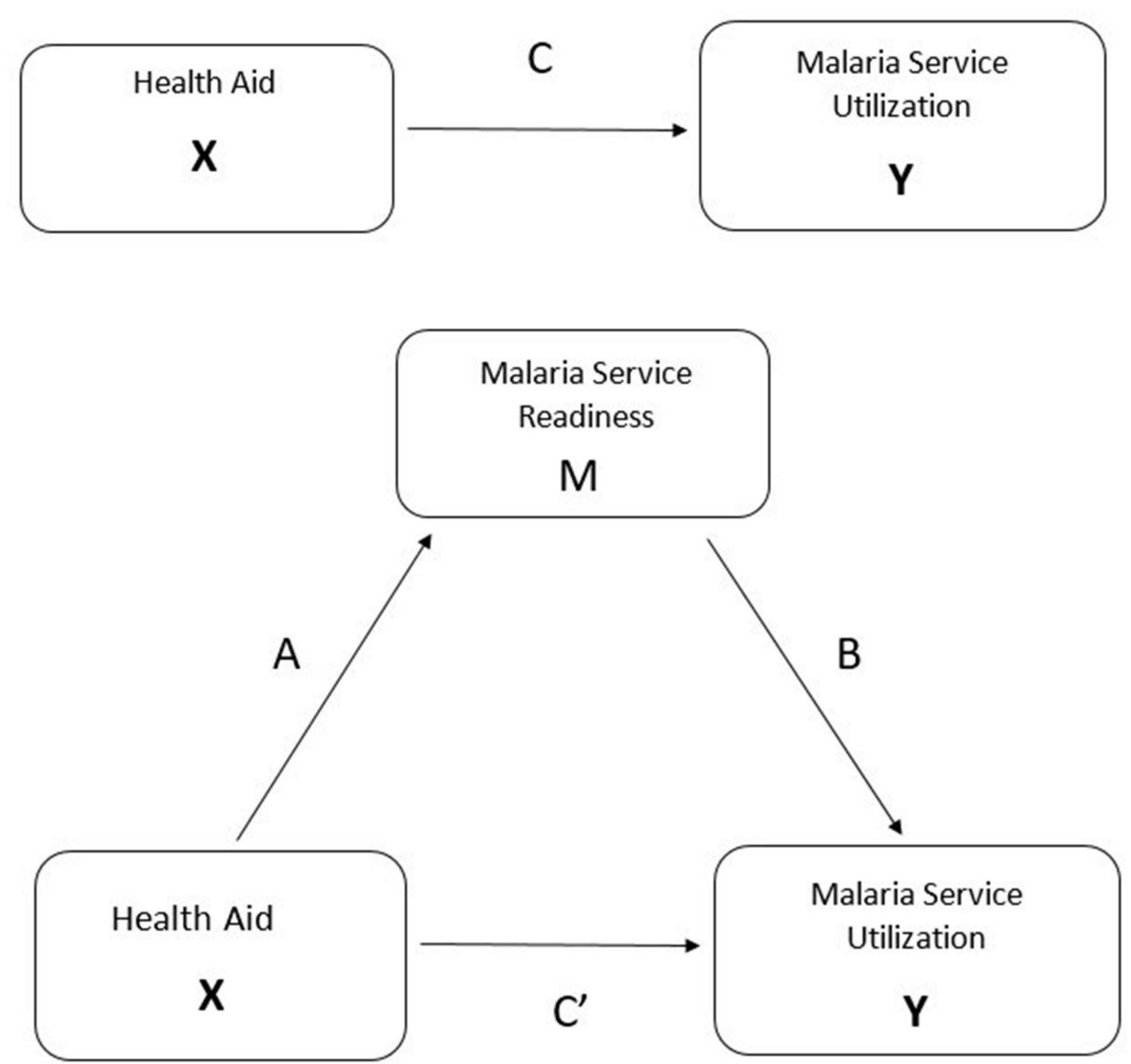

Figure 1 Mediation model relating health aid, malaria service readiness and utilisation.

representing the time period before the Service Provision Assessment (SPA) was completed 2005-2012. This variable was subset from AidData's Malawi Aid Management Platform (AMP), including data on the locations of aid projects recorded by the Government of Malawi's AMP and includes roughly $80 \%$ of all aid reported to the Ministry of Finance since 2000. Data in Malawi's AMP are hierarchically coded, where purpose codes represent the overarching purpose of aid projects and activity codes are subcategories of purpose codes indicating specific project activities. Health aid includes only projects that could promote malaria services and utilisation locally, including those with a purpose or activity code from the following aid sectors: basic healthcare, basic health infrastructure, health policy and administrative management, infectious and parasitic disease control, medical education and training or medical services. ${ }^{9}{ }^{10}$ In addition, each aid project carries a precision code that indicates the spatial certainty of where the project was allocated. I include all projects with a spatial precision at the district level, excluding projects with spatial precision at the region and country level. This eliminated 27 project locations, leaving 108 in the sample. Projects allocated to a specific location are coded as being allocated to a geographic region, specifically the first-level administrative division, the largest administrative subdivision of a country. Children were coded as living in an area that received aid based on the count of health aid projects allocated to the region in which they resided during the survey. The aid data, while granular, do not distinguish which survey respondents received aid. Therefore, estimates refer to individuals' residence in areas that received aid.

\section{Index of malaria service readiness}

The second key independent variable is an index of malaria service readiness, a quality measure of facilities' capacity to provide malaria services. Using the Service Availability and Readiness Assessment as a framework and data representing responses to 40 variables included in the 2013/2014 Malawi SPA, an index of malaria service readiness was created using a weighted additive approach ${ }^{11}$ These 40 variables are categorised into six groups that are outlined in online supplementary appendix E. An illustration of the construction of the total malaria service readiness index is available in online supplementary appendix E. A facility was given one point for reporting yes to each category, producing malaria service readiness scores ranging from 0 to 6 . The third and fourth independent variables of interest are a subset of the total malaria service readiness index. Facility readiness for diagnostic capacity combines indicators from rows 1, 2 and 3 (online supplementary 
Table 1 Malaria treatment in facilities offering curative care to children (\%)

\begin{tabular}{|c|c|c|c|c|c|c|c|c|}
\hline & $\begin{array}{l}(1) \\
\text { Either } \\
\text { malaria } \\
\text { RDT or } \\
\text { malaria } \\
\text { microscopy } \\
\text { diagnostics }\end{array}$ & $\begin{array}{l}(2) \\
\text { Either } \\
\text { malaria } \\
\text { RDT or } \\
\text { malaria } \\
\text { microscopy } \\
\text { training }\end{array}$ & $\begin{array}{l}\text { (3) } \\
\text { Malaria } \\
\text { RDT } \\
\text { protocol }\end{array}$ & $\begin{array}{l}(4) \\
\text { Malaria } \\
\text { treatment } \\
\text { guidelines }\end{array}$ & $\begin{array}{l}(5) \\
\text { First-line } \\
\text { treatment } \\
\text { medicine }\end{array}$ & $\begin{array}{l}\text { (6) } \\
\text { Trained } \\
\text { personnel }\end{array}$ & $\begin{array}{l}(7) \\
\text { Malaria } \\
\text { diagnostic } \\
\text { capacity }\end{array}$ & $\begin{array}{l}(8) \\
\text { Malaria } \\
\text { service } \\
\text { readiness } \\
\text { index }\end{array}$ \\
\hline \multicolumn{9}{|l|}{ Facility type } \\
\hline Hospital & 99 & 77 & 76 & 73 & 99 & 79 & 54 & 42 \\
\hline Health centre & 99 & 61 & 67 & 71 & 100 & 56 & 43 & 29 \\
\hline Dispensary & 95 & 37 & 49 & 51 & 91 & 36 & 20 & 12 \\
\hline \multicolumn{9}{|c|}{ Managing authority } \\
\hline Government & 97 & 62 & 67 & 70 & 96 & 59 & 45 & 31 \\
\hline CHAM & 100 & 58 & 68 & 72 & 100 & 54 & 39 & 29 \\
\hline Private & 97 & 37 & 42 & 47 & 95 & 36 & 17 & 9 \\
\hline $\begin{array}{l}\text { Mission/Faith- } \\
\text { based }\end{array}$ & 100 & 71 & 57 & 43 & 100 & 71 & 43 & 29 \\
\hline NGO & 96 & 53 & 60 & 55 & 96 & 47 & 33 & 16 \\
\hline Company & 100 & 31 & 49 & 54 & 95 & 35 & 20 & 11 \\
\hline \multicolumn{9}{|l|}{ Region } \\
\hline Northern & 99 & 51 & 69 & 65 & 98 & 49 & 38 & 24 \\
\hline Central East & 99 & 64 & 65 & 70 & 99 & 64 & 45 & 35 \\
\hline Central West & 96 & 59 & 53 & 59 & 95 & 59 & 35 & 24 \\
\hline South East & 99 & 52 & 54 & 64 & 97 & 44 & 32 & 22 \\
\hline South West & 97 & 45 & 63 & 62 & 95 & 44 & 32 & 18 \\
\hline Total & 98 & 53 & 60 & 63 & 96 & 51 & 35 & 24 \\
\hline
\end{tabular}

Note: Hospitals include central, district, rural and other hospitals; health centres include maternity centres and dispensaries include clinic and health posts. (1) Interviewer observed RDT being conducted OR providers in facility diagnose malaria OR providers in the facility prescribe treatment for malaria; facility had malaria microscopy capacity defined as a facility had functioning microscope with glass slides and relevant stains for malaria microscopy available somewhere in the facility; facility had RDT capacity defined as the facility having an unexpired malaria RDT kit available somewhere in the facility. (2) Facility had at least one interviewed provider of child curative care services who reported receiving in-service training on malaria RTD or malaria microscopy during the 24 months preceding the assessment. The training must have involved structured sessions; it does not include individual instruction that a provider might have received during routine supervision. (3) Training manual, poster or other job aid for using malaria RDT observed OR reported. (4) National OR other guidelines for the diagnosis and treatment of malaria in the facility service area observed. (5) Facility had at least one of the following valid, antimalarial medicines available: artemether lumefantrine: 6,12,18 OR 24 tablets, Fansidar, quinine tablets, quinine injection, injectable artesunate, artesunate suppositories, other antimalaria medicine, artemether-amodiaquine $25 \mathrm{mg}, 50 \mathrm{mg}$ OR $100 \mathrm{mg}$. (6) Facility had at least one interviewed provider of child curative care services who reported receiving in-service training in: diagnosis malaria in adults OR children, how to perform malaria RDT, treatment of malaria in adults, treatment of malaria during pregnancy, intermittent preventive treatment of malaria in pregnancy, treatment of malaria in children. (7) Facility had either malaria RDT or microscopy, either malaria RDT or microscopy training AND malaria RDT protocol. (8) Facility had malaria RDT or microscopy, either malaria RDT or microscopy training, malaria treatment guidelines, first-line treatment medicine AND trained personnel. $\mathrm{RDT}$, rapid diagnostic test.

appendix E) that include malaria rapid diagnostic testing (RDT) or malaria microscopy diagnostics, either malaria RDT training or malaria microscopy training, and having malaria RDT protocols. Facility readiness for malaria training includes indicators from rows 2, 3, 4 and 6 from online supplementary appendix E: having either malaria RDT or malaria microscopy training, malaria RDT protocols, malaria treatment guidelines and trained personnel. Kernel density estimation (KDE) was used to link the malaria service readiness index to the household indirectly in order to create the index of malaria service readiness. Appendices $\mathrm{E}$ and $\mathrm{F}$ provide, respectively, further details on construction of the malaria service readiness index and the link $(\mathrm{F})$ between facility-level and household-level data.

\section{RESULTS}

\section{Summary statistics}

Among the 956 facilities in Malawi in 2013, 49\% were health centres, followed by dispensaries (39\%) and hospitals $(12 \%)$. The availability of malaria services 
Table 2 Logistic regression models to investigate the association between health aid and utilisation $(n=2118)$

\begin{tabular}{|c|c|c|c|c|}
\hline \multirow[b]{3}{*}{ Variables } & (1) & (2) & (3) & (4) \\
\hline & Advice or treatment & Heel/Finger stick & Receiving ACT & Any malaria services \\
\hline & OR (95\% Cl) & OR $(95 \% \mathrm{Cl})$ & OR $(95 \% \mathrm{Cl})$ & OR $(95 \% \mathrm{Cl})$ \\
\hline $\begin{array}{l}\text { Health aid (project } \\
\text { count) }\end{array}$ & $0.957(0.900$ to 1.018$)$ & 0.967 (0.882 to 1.060$)$ & $1.001(0.933$ to 1.073$)$ & $0.976(0.920$ to 1.035$)$ \\
\hline Night-time lights & 0.989 (0.848 to 1.152$)$ & 1.029 (0.828 to 1.277$)$ & 1.092 (0.904 to 1.319$)$ & 0.974 (0.838 to 1.132$)$ \\
\hline Population & $1.000^{\star *}(1.000$ to 1.000$)$ & $1.000^{* *}(1.000-1.000)$ & $1.000(1.000$ to 1.000$)$ & $1.000^{\star \star}(1.000$ to 1.000$)$ \\
\hline Area (square km) & $1.009^{\star \star}(1.001$ to 1.017$)$ & $1.016^{\star \star \star}(1.005$ to 1.028$)$ & 1.008 (0.998 to 1.019$)$ & $1.007^{\star}(0.999$ to 1.015$)$ \\
\hline \multicolumn{5}{|l|}{ Mother's education } \\
\hline Primary & $1.255(0.890$ to 1.771$)$ & 1.555 (0.894 to 2.704$)$ & 1.239 (0.824 to 1.865$)$ & 1.294 (0.923 to 1.813$)$ \\
\hline Secondary & $1.531^{*}(0.978$ to 2.395$)$ & $2.043^{\star *}(1.041$ to 4.012$)$ & 1.509 (0.873 to 2.610$)$ & $1.558^{\star \star}(1.004$ to 2.417$)$ \\
\hline Higher & $0.834(0.180$ to 3.871$)$ & 2.406 (0.478 to 12.097$)$ & 0.941 (0.116 to 7.618$)$ & 0.829 (0.179 to 3.841$)$ \\
\hline Age of mother & $1.004(0.985$ to 1.023$)$ & $0.996(0.968$ to 1.025$)$ & $1.004(0.981$ to 1.028$)$ & $0.996(0.978$ to 1.015$)$ \\
\hline Age of child & $0.892^{\star \star}(0.817$ to 0.974$)$ & $0.890^{*}(0.783$ to 1.012$)$ & 0.968 (0.871 to 1.075$)$ & $0.904^{\star *}(0.829$ to 0.984$)$ \\
\hline $\begin{array}{l}\text { Distance from nearest } \\
\text { Health Facility }\end{array}$ & 0.957 (0.906 to 1.010$)$ & $0.921^{*}(0.845$ to 1.003$)$ & 0.953 (0.892 to 1.017$)$ & 0.957 (0.907 to 1.009$)$ \\
\hline \multicolumn{5}{|l|}{ Wealth Index } \\
\hline Poorer & 1.304 (0.889 to 1.911$)$ & $1.062(0.612$ to 1.844$)$ & 0.961 (0.620 to 1.488$)$ & $1.280(0.883$ to 1.854$)$ \\
\hline Middle & 0.982 (0.658 to 1.465$)$ & 0.704 (0.386 to 1.287$)$ & $0.666^{\star}(0.414$ to 1.071$)$ & 0.961 (0.652 to 1.416$)$ \\
\hline Richer & 1.135 (0.764 to 1.685$)$ & 0.778 (0.435 to 1.392$)$ & 0.939 (0.599 to 1.471$)$ & 1.129 (0.769 to 1.658$)$ \\
\hline Richest & 1.095 (0.688 to 1.741$)$ & 0.795 (0.414 to 1.526$)$ & 0.695 (0.393 to 1.229$)$ & 1.081 (0.687 to 1.702$)$ \\
\hline \multicolumn{5}{|l|}{ Geographic classification } \\
\hline Rural & 1.002 (0.668 to 1.502$)$ & 0.694 (0.401 to 1.200$)$ & 1.442 (0.858 to 2.425$)$ & 1.136 (0.761 to 1.697$)$ \\
\hline Malaria Ecology Index & $2.589^{\star \star \star}(1.577$ to 4.248$)$ & $2.273^{\star \star}(1.112$ to 4.646$)$ & $2.074^{\star \star}(1.139$ to 3.775$)$ & $2.496^{\star \star *}(1.535$ to 4.056$)$ \\
\hline Constant & $0.062^{\star * \star}(0.021$ to 0.177$)$ & $0.024^{\star \star \star}(0.005$ to 0.113$)$ & $0.037^{\star \star \star}(0.010$ to 0.134$)$ & $0.077^{\star * *}(0.027$ to 0.215$)$ \\
\hline
\end{tabular}

Reference group includes no education, poorest and urban geographic location.

${ }^{\star * * *} \mathrm{P}<0.01,{ }^{* *} \mathrm{P}<0.05,{ }^{\star} \mathrm{P}<0.1$.

$\mathrm{ACT}$, artemisinin-based combination no education, poorest and urban geographic location.

varied by type of facility, with hospitals and health centres generally having many of the indicators comprising the malaria service readiness index and dispensaries having few (table 1). Common across all facility types were diagnostic capacity and the availability of first-line medicine to treat malaria; having a staff member recently trained in RDT or microscopy was less common, especially in dispensaries. Hospitals and health centres were similar in the availability of services, except that $79 \%$ of hospitals and only $56 \%$ of health centres had personnel recently trained in malaria diagnosis and/or treatment. Most dispensaries had RDT and microscopy diagnostics (95\%) and first-line medicine $(91 \%)$, but few had other types of malaria services. Less than one-third of government, Christian Health Association of Malawi and mission/ faith-based facilities are malaria-service ready, but only $9 \%$ of privately managed facilities are. Readiness was lowest in the southwest (18\%) and highest in the central east $(35 \%)$. Overall, only $35 \%$ of facilities had full diagnostic capacity.

Of the 2171 children under five in the sample, $17 \%$ received any malaria services, with $7 \%$ receiving a finger or heel stick and 10\% receiving ACT. Online supplementary appendix $\mathrm{H}$ provides descriptive statistics of the individual determinants based on predisposing, enabling and need factors from the conceptual model. Most $(75 \%)$ of the children in the sample live in rural locations; they were equally distributed across years of age and levels of household wealth and most $(71 \%)$ lived more than $2 \mathrm{~km}$ from a facility. The mean level of the Malaria Ecology Index was 0.310 and was similar in both urban and rural locations with a mean level of risk of 0.274 in urban locations and 0.312 in rural location, consistent with endemic levels of malaria risk across Malawi.

\section{Adjusted associations between health aid and utilisation (Step 1: Pathway C)}

Table 2 presents ORs for the adjusted association between health aid and malaria service utilisation. Row 1 presents the adjusted association of health aid across malaria utilisation outcomes and suggests that health aid has a negative or no association with children's use of malaria services, but these associations are not significant. 
Table 3 Results of multinomial Logit regression of health aid on average readiness to deliver malaria services $(n=2118)$

Overall malaria service Malaria diagnostic readiness readiness index

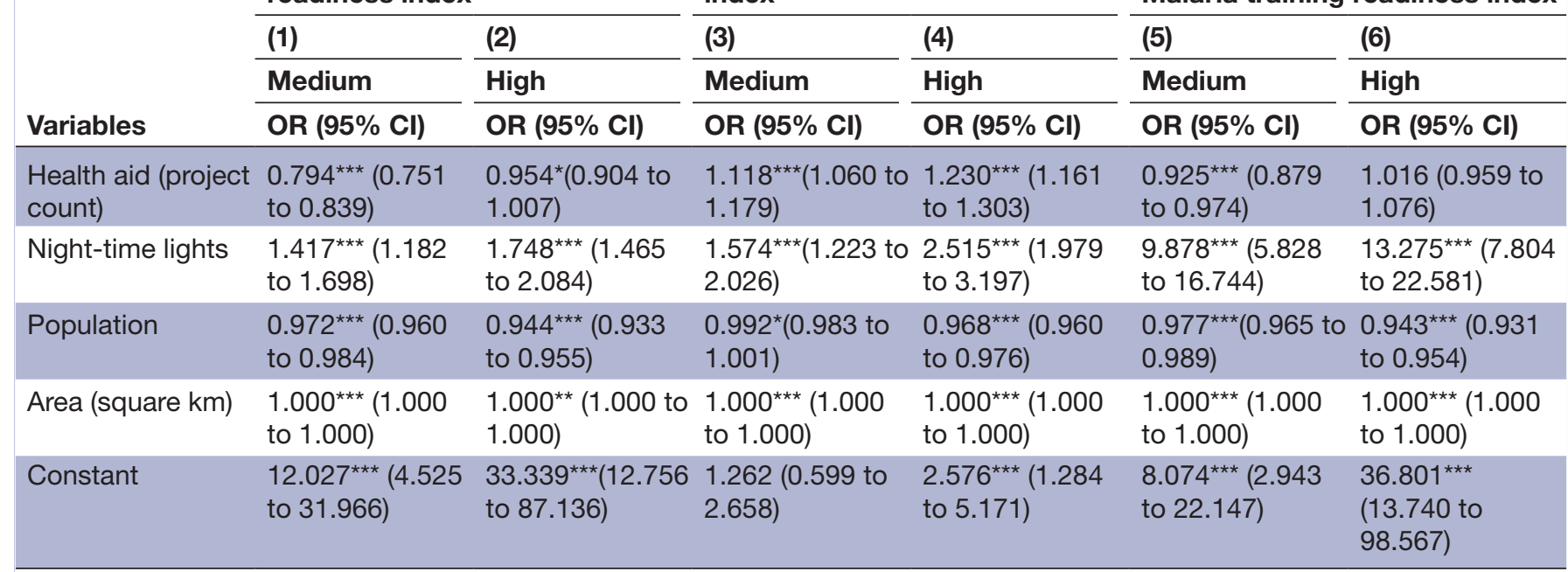

Reference group includes low malaria service readiness, low malaria diagnostic readiness, low malaria training readiness.

${ }^{* \star * \star} \mathrm{P}<0.01,{ }^{* *} \mathrm{P}<0.05,{ }^{*} \mathrm{P}<0.1$.

$\mathrm{ACT}$, artemisinin-based combination therapy.

Using heel stick (Column 2) as an example, the odds $(\mathrm{OR}=0.967 ; 95 \% \mathrm{CI} 0.882$ to 1.060$)$ of receiving a heel or finger stick are $4 \%$ lower for each additional health aid project in the region.

\section{Adjusted associations between health aid and facility readiness to deliver malaria services (Step 2: Pathway A)}

Using a multinomial logistic regression model, table 3 presents results in the form of relative risk (RR) ratios, testing adjusted associations between health aid and overall malaria service readiness as well as for diagnostic and training readiness. Columns 1 and 2 present overall facility readiness to deliver malaria services and indicate that increasing the number of aid projects in a region is associated with statistically significant lower levels of malaria readiness. This is also true for malaria training readiness (Columns 5 and 6), but not malaria diagnostic readiness (Columns 3 and 4), where results indicate a positive relationship between increasing aid and diagnostic capacity. More specifically, examining overall malaria service readiness (Column 1) shows that if health aid to a region was to increase by one project, the RR for medium readiness relative to low readiness would likely decrease by a factor of 0.794 (95\% CI 0.751 to 0.839$)$. More generally, we can say that if health aid increases in a region by one project, the RR of having a medium level of readiness instead of a low level of readiness likely would decrease by $21 \%$. An additional aid project also decreases high levels of facility readiness $(\mathrm{RR}=0.954 ; 95 \%$ CI 0.904 to 1.007 ).

For readiness to provide diagnostic services, results indicate a positive association between aid and diagnostic capacity (Columns 3 and 4). Results indicate that the relative likelihood that a facility has a medium level of diagnostic readiness increases by $12 \%$ ( $R R=1.118 ; 95 \%$ CI 1.060 to 1.179) and the relative likelihood that a facility has a high level of readiness increases by $23 \%$ ( $R R=1.230 ; 95 \%$ CI 1.161 to 1.303$)$. However, malaria training readiness results (Columns 5 and 6) indicate a negative association between aid and training readiness. Specifically, an additional aid project in a region does not increase facilities' likelihood of having a high level of training readiness $(\mathrm{RR}=1.016 ; 95 \%$ CI 0.959 to 1.076 ) and actually decreases their relative likelihood of having a medium level of training readiness by $8 \%(\mathrm{RR}=0.925 ; 95 \%$ CI 0.879 to 0.974$)$.

\section{Adjusted associations between health aid, malarial readiness and utilisation(Steps 3 and 4: Pathways B and C')}

Tables $4 \mathrm{~A}-\mathrm{C}$ are one table split into three tables for clarity. They indicate that when both malaria service readiness and aid were taken into account, there is little association with utilisation. That is, results show an insignificant, though positive, correlation between facilities with a medium level of overall diagnostic capacity and use of malaria services for children. Results indicate a weak significant association $(\mathrm{p}<0.10)$ and the odds of children receiving a heel or finger stick was $59 \%$ higher in facilities with a medium level of readiness compared with low readiness facilities (OR=1.598; 95\% CI 0.915 to 2.793). However, this weak association was not apparent in facilities with a high level of readiness. Among facilities with a high level of readiness the odds of receiving ACT was $45 \%$ lower than in those with low levels of readiness $(\mathrm{OR}=0.559,95 \%$ CI 0.310 to 1.007$)$, but again, this association was weak $(p<0.10)$. Turning to the other independent variable of interest, health aid, results showing a negative association between health aid and all utilisation outcomes are also not statistically significant.

\section{DISCUSSION}

This research uses a mediation approach, disaggregated data on health aid and data from nationally representative 
Table 4A Logistic regression models to investigate the association between overall malaria service readiness, health aid and utilisation $(n=2118)$

\begin{tabular}{|c|c|c|c|c|}
\hline \multirow[b]{4}{*}{ Variables } & \multicolumn{4}{|c|}{ Overall malaria service readiness } \\
\hline & (1) & \multirow{2}{*}{$\begin{array}{l}\text { (2) } \\
\text { Hee }\end{array}$} & (3) & (4) \\
\hline & Advice or treatment & & Receiving ACT & Any malaria services \\
\hline & OR $(95 \% \mathrm{Cl})$ & OR $(95 \% \mathrm{Cl})$ & OR $(95 \% \mathrm{Cl})$ & OR $(95 \% \mathrm{Cl})$ \\
\hline \multicolumn{5}{|c|}{ Overall malaria service readiness } \\
\hline Medium & $1.100(0.767$ to 1.576$)$ & $1.598^{\star}(0.915$ to 2.793$)$ & $0.952(0.625$ to 1.451$)$ & $1.089(0.768$ to 1.545$)$ \\
\hline High & $0.816(0.502$ to 1.329$)$ & $1.060(0.500$ to 2.246$)$ & $0.559^{*}(0.310$ to 1.007$)$ & $0.830(0.519$ to 1.328$)$ \\
\hline $\begin{array}{l}\text { Health aid } \\
\text { (project count) }\end{array}$ & $0.963(0.904$ to 1.027$)$ & $0.988(0.898$ to 1.087$)$ & $1.004(0.933$ to 1.080$)$ & $0.982(0.923$ to 1.043$)$ \\
\hline Night-time lights & $0.992(0.850$ to 1.157$)$ & $1.026(0.824$ to 1.277$)$ & $1.104(0.913$ to 1.336$)$ & $0.977(0.840$ to 1.137$)$ \\
\hline Population & $1 \cdot 000^{\star \star}(1 \cdot 000$ to $1 \cdot 000)$ & $1.000 *(1.000$ to $1 \cdot 000)$ & $1.000(1.000$ to 1.000$)$ & $1 \cdot 000^{\star \star}(1.000$ to 1.000$)$ \\
\hline \multicolumn{5}{|l|}{ Area (square km) } \\
\hline & $1.009^{* \star}(1.001$ to 1.017$)$ & $1 \cdot 017^{\star \star \star}(1.005$ to $1 \cdot 028)$ & $1.009(0.998$ to 1.019$)$ & $1.007^{\star}(0.999$ to 1.015$)$ \\
\hline \multicolumn{5}{|c|}{ Mother's education } \\
\hline Primary & $1 \cdot 257(0.890$ to 1.775$)$ & $1.573(0.904$ to 2.737$)$ & $1 \cdot 234(0 \cdot 819$ to $1 \cdot 860)$ & $1.296(0.924$ to 1.817$)$ \\
\hline Secondary & $1.527^{\star}(0.975$ to 2.392$)$ & $2 \cdot 045^{\star \star}(1.039$ to 4.028$)$ & $1.503(0.867$ to $2 \cdot 607)$ & $1 \cdot 555^{\star \star}(1 \cdot 001$ to $2 \cdot 414)$ \\
\hline Higher & $0.840(0.181$ to 3.911$)$ & $2 \cdot 471(0 \cdot 490$ to $12 \cdot 468)$ & $0.935(0.115$ to $7 \cdot 616)$ & $0.835(0.180$ to 3.876$)$ \\
\hline Age of mother & $1.004(0.985$ to 1.023$)$ & $0.996(0.968$ to 1.025$)$ & $1.004(0.981$ to 1.028$)$ & $0.996(0.978$ to 1.015$)$ \\
\hline Age of child & $0.895^{\star \star}(0.820$ to 0.977$)$ & $0.896^{*}(0.787$ to 1.019$)$ & $0.973(0.875$ to 1.082$)$ & $0.906^{\star \star}(0.832$ to 0.988$)$ \\
\hline $\begin{array}{l}\text { Distance from } \\
\text { nearest Health } \\
\text { Facility }\end{array}$ & $0.953(0.889$ to 1.021$)$ & $0.947(0.852$ to 1.053$)$ & $0.926^{\star}(0.853$ to 1.006$)$ & $0.953(0.891$ to 1.019$)$ \\
\hline \multicolumn{5}{|l|}{ Wealth Index } \\
\hline Poorer & $\begin{array}{l}1.311 \\
(0.894-1.922)\end{array}$ & $\begin{array}{l}1 \cdot 070 \\
(0 \cdot 616-1 \cdot 859)\end{array}$ & $\begin{array}{l}0.970 \\
(0.626-1.503)\end{array}$ & $\begin{array}{l}1 \cdot 286 \\
(0 \cdot 887-1 \cdot 864)\end{array}$ \\
\hline Middle & $\begin{array}{l}0.990 \\
(0.663 \text { to } 1.479)\end{array}$ & $\begin{array}{l}0.724 \\
(0.396 \text { to } 1.324)\end{array}$ & $\begin{array}{l}0.667^{*} \\
(0.415 \text { to } 1.073)\end{array}$ & $\begin{array}{l}0.969 \\
(0.657 \text { to } 1.429)\end{array}$ \\
\hline Richer & 1.147 (0.772 to 1.704$)$ & 0.786 (0.439 to 1.408$)$ & 0.960 (0.612 to 1.506$)$ & 1.141 (0.777 to 1.677$)$ \\
\hline Richest & $\begin{array}{l}1.094 \\
(0.697 \text { to } 1.765)\end{array}$ & $\begin{array}{l}0.698 \\
(0.434 \text { to } 1.604)\end{array}$ & $\begin{array}{l}0.835 \\
(0.395 \text { to } 1.236)\end{array}$ & $\begin{array}{l}1 \cdot 110 \\
(0.694 \text { to } 1 \cdot 723)\end{array}$ \\
\hline \multicolumn{5}{|c|}{ Geographic classification } \\
\hline Rural & $0.848(0.535$ to 1.342$)$ & $0.568^{*}(0.303$ to 1.065$)$ & $1.038(0.573$ to $1 \cdot 880)$ & $0.976(0.621$ to 1.535$)$ \\
\hline $\begin{array}{l}\text { Malaria Ecology } \\
\text { Index }\end{array}$ & $2 \cdot 606^{\star \star \star}(1.583$ to $4 \cdot 290)$ & $2 \cdot 319^{\star \star}(1 \cdot 116$ to $4 \cdot 818)$ & $2 \cdot 091^{\star \star}(1 \cdot 141$ to $3 \cdot 830)$ & $2.507^{\star \star \star}(1.538$ to 4.087$)$ \\
\hline Constant & $0.070^{\star \star \star}(0.021$ to 0.233$)$ & $0.018^{\star \star \star}(0.003$ to 0.108$)$ & $0.064^{\star \star \star}(0.015$ to 0.281$)$ & $0.087^{\star \star \star}(0.027$ to 0.282$)$ \\
\hline
\end{tabular}

Reference group includes low malaria service readiness, low malaria diagnostic readiness, low malaria training readiness, no education, poorest and urban geographic location.

${ }^{* * *} \mathrm{P}<0.01,{ }^{* *} \mathrm{P}<0.05,{ }^{*} \mathrm{P}<0.1$

$\mathrm{ACT}$, artemisinin-based combination therapy.

household and health facility surveys and links malaria facility information to malaria service use at the child level. While existing literature has examined the effect of facility readiness to provide contraceptive services as well as facility readiness to provide care during delivery, these studies do not consider the role of health aid. ${ }^{12} 13$ Further, there is a considerable lack of knowledge on the effect of health aid on the readiness of facilities to provide malaria services and on malaria service utilisation in sub-Saharan Africa. This study sought to inform aid allocation strategies designed to strengthen malaria service delivery in Malawi using a theoretically driven health services approach, but found limited evidence that health aid relates to malaria health service utilisation through its influence on increasing malaria facility readiness in a defined service area. Despite this finding, results did reveal that health aid increased diagnostic capacity within health facilities. The finding that health aid does contribute to the availability of diagnostic tools, but not an overall ability to implement these tools indicates 
Table 4B Logistic regression models to investigate the association between malaria diagnostic service readiness, health aid and utilisation $(n=2118)$

\begin{tabular}{|c|c|c|c|c|}
\hline \multirow[b]{4}{*}{ Variables } & \multicolumn{4}{|c|}{ Malaria diagnostic readiness } \\
\hline & (1) & (2) & (3) & $(4)$ \\
\hline & Advice or treatment & Heel/Finger stick & Receiving ACT & Any malaria services \\
\hline & OR $(95 \% \mathrm{Cl})$ & OR $(95 \% \mathrm{Cl})$ & OR $(95 \% \mathrm{Cl})$ & OR $(95 \% \mathrm{Cl})$ \\
\hline \multicolumn{5}{|c|}{ Malaria diagnosis readiness } \\
\hline Medium & $1.182(0.855$ to 1.633$)$ & $1.299(0.806$ to 2.091$)$ & $1.080(0.733$ to 1.591$)$ & $1.134(0.827$ to 1.554$)$ \\
\hline High & $1.066(0.712$ to 1.597$)$ & $0.809(0.443$ to 1.477$)$ & $0.912(0.557$ to 1.491$)$ & $1.048(0.707$ to 1.554$)$ \\
\hline $\begin{array}{l}\text { Health aid (project } \\
\text { count) }\end{array}$ & $0.956(0.899$ to 1.018$)$ & $0.972(0.886$ to 1.066$)$ & $1.003(0.935$ to 1.077$)$ & $0.975(0.919$ to 1.035$)$ \\
\hline Night-time lights & $0.989(0.837$ to 1.167$)$ & $1.094(0.865$ to $1 \cdot 383)$ & $1.119(0.911$ to 1.374$)$ & $0.974(0.828$ to $1 \cdot 147)$ \\
\hline Population & $1 \cdot 000^{\star *}(1 \cdot 000$ to $1 \cdot 000)$ & $1 \cdot 000^{*}(1.000$ to $1 \cdot 000)$ & $1.000(1.000$ to 1.000$)$ & $1 \cdot 000^{* \star}(1.000$ to $1 \cdot 000)$ \\
\hline Area (square km) & $1 \cdot 008^{\star *}(1 \cdot 000$ to $1 \cdot 017)$ & $1.016^{\star * \star}(1.004$ to 1.027$)$ & $1.008(0.997$ to 1.019$)$ & $1.007(0.999$ to 1.014$)$ \\
\hline \multicolumn{5}{|l|}{ Mother's education } \\
\hline Primary & $1 \cdot 238(0.876$ to $1 \cdot 749)$ & $1.518(0.870$ to $2 \cdot 649)$ & $1 \cdot 234(0.818$ to $1 \cdot 861)$ & $1.280(0.912$ to 1.796$)$ \\
\hline Secondary & $1.515^{\star}(0.967$ to $2 \cdot 374)$ & $2 \cdot 047^{\star \star}(1.039$ to 4.033$)$ & $1.516(0.874$ to $2 \cdot 628)$ & $1.545^{\star}(0.994$ to $2 \cdot 401)$ \\
\hline Higher & $0.823(0.177$ to $3 \cdot 826)$ & $2.462(0.490$ to 12.374$)$ & $0.949(0.117$ to 7.693$)$ & $0.821(0.177$ to 3.807$)$ \\
\hline Age of mother & $1.004(0.985$ to 1.024$)$ & $0.997(0.969$ to 1.027$)$ & $1.005(0.982$ to 1.028$)$ & $0.997(0.978$ to 1.016$)$ \\
\hline Age of child & $0.891^{\star *}(0.817$ to 0.973$)$ & $0.890^{*}(0.782$ to 1.012$)$ & $0.968(0.871$ to 1.076$)$ & $0.903^{\star *}(0.829$ to 0.984$)$ \\
\hline $\begin{array}{l}\text { Distance from } \\
\text { nearest Health } \\
\text { Facility }\end{array}$ & $0.966(0.907$ to 1.028$)$ & $0.920^{*}(0.836$ to 1.014$)$ & $0.951(0.882$ to 1.025$)$ & $0.964(0.907$ to 1.024$)$ \\
\hline \multicolumn{5}{|l|}{ Wealth Index } \\
\hline Poorer & $1.306(0.891$ to 1.914$)$ & $1.076(0.619$ to $1 \cdot 870)$ & $0.962(0.621$ to 1.489$)$ & $1 \cdot 281(0.884$ to $1 \cdot 856)$ \\
\hline Middle & $0.980(0.656$ to 1.462$)$ & $0.718(0.393$ to 1.314$)$ & $0.667^{\star}(0.415$ to $1 \cdot 074)$ & $0.959(0.650$ to 1.415$)$ \\
\hline Richer & $1.138(0.766$ to $1 \cdot 689)$ & $0.788(0.441$ to 1.410$)$ & $0.941(0.600$ to 1.474$)$ & $1 \cdot 131(0.771$ to $1 \cdot 661)$ \\
\hline Richest & $1 \cdot 102(0.693$ to 1.753$)$ & $0.819(0.426$ to 1.574$)$ & $0.697(0.394$ to 1.234$)$ & $1.086(0.690$ to 1.711$)$ \\
\hline \multicolumn{5}{|c|}{ Geographic classification } \\
\hline Rural & $0.977(0.649$ to 1.470$)$ & $0.641(0.370$ to $1 \cdot 113)$ & $1.411(0.837$ to $2 \cdot 377)$ & $1 \cdot 115(0.745$ to $1 \cdot 671)$ \\
\hline $\begin{array}{l}\text { Malaria Ecology } \\
\text { Index }\end{array}$ & $2 \cdot 553^{\star \star \star}(1.540$ to 4.235$)$ & $2.030^{*}(0.981$ to $4 \cdot 199)$ & $1.979^{\star \star}(1 \cdot 071$ to $3 \cdot 655)$ & $2 \cdot 470^{\star \star \star}(1.504$ to $4 \cdot 055)$ \\
\hline Constant & $0.057^{\star \star \star}(0.018$ to 0.177$)$ & $0.028^{\star \star \star}(0.005$ to $0 \cdot 148)$ & $0.041^{\star \star \star}(0.010$ to 0.167$)$ & $0.073^{\star \star \star}(0.024$ to 0.220$)$ \\
\hline
\end{tabular}

Reference group includes low malaria service readiness, low malaria diagnostic readiness, low malaria training readiness, no education, poorest and urban geographic location.

${ }^{\star * \star \star *} \mathrm{P}<0.01,{ }^{\star \star} \mathrm{P}<0.05,{ }^{\star} \mathrm{P}<0.1$.

$\mathrm{ACT}$, artemisinin-based combination therapy.

a clear gap for funders to consider when developing future allocation policies. This section will first discuss the results by each of the four research questions, then discuss study limitations and strengths and conclude with the contributions of this research.

\section{Discussion of results}

According to the Baron and Kenney mediation approach, Step 1 typically would necessitate a significant association between health aid and malaria service utilisation to justify continuing the mediation analysis. In recent years, the literature has supported dropping the Step 1 requirement that Pathway $\mathrm{C}$ or the 'total effect' is significant to assess mediation. ${ }^{14}$ Recent studies have demonstrated that mediation analyses provide explanatory value and should be pursued even when the total effect is not statistically significant. ${ }^{14-16}$ In addition, if the 'total effect' of health aid on utilisation of malaria services is not significant, there may be practical or theoretical reasons for estimating mediating effects. ${ }^{17} \mathrm{In}$ the case of this study, identifying the association between health aid and malaria service readiness still has scientific merit in order to understand which components of the malaria healthcare system need to be strengthened or require improved measurement. ${ }^{17}$ Referring to the Baron and Kenney model, results from Step 2 indicate a significant relationship between aid and diagnostic service readiness, but not overall malaria readiness or training readiness. 
Table 4C Logistic regression models to investigate the association between malaria training service readiness, health aid and utilisation $(n=2118)$

\begin{tabular}{|c|c|c|c|c|}
\hline \multirow[b]{4}{*}{ Variables } & \multicolumn{4}{|c|}{ Malaria training service readiness } \\
\hline & (1) & \multirow{2}{*}{$\begin{array}{l}\text { (2) } \\
\text { Heel/Finger stick }\end{array}$} & \multirow{2}{*}{$\begin{array}{l}\text { (3) } \\
\text { Receiving ACT }\end{array}$} & (4) \\
\hline & Advice or treatment & & & Any malaria services \\
\hline & OR $(95 \% \mathrm{Cl})$ & OR $(95 \% \mathrm{Cl})$ & OR $(95 \% \mathrm{Cl})$ & OR $(95 \% \mathrm{Cl})$ \\
\hline \multicolumn{5}{|c|}{ Malaria training readiness } \\
\hline Medium & $1 \cdot 259(0.878$ to $1 \cdot 804)$ & $1.433(0.820$ to 2.506$)$ & $1.137(0.743$ to $1 \cdot 739)$ & $1 \cdot 263(0.890$ to $1 \cdot 791)$ \\
\hline High & $0.852(0.504$ to 1.440$)$ & $1 \cdot 197(0.548$ to $2 \cdot 617)$ & $0.728(0.390$ to 1.359$)$ & $0.846(0.509$ to 1.408$)$ \\
\hline $\begin{array}{l}\text { Health aid (project } \\
\text { count) }\end{array}$ & $0.964(0.906$ to 1.026$)$ & $0.972(0.887$ to 1.066$)$ & $1.007(0.939$ to 1.081$)$ & $0.983(0.926$ to 1.043$)$ \\
\hline Night-time lights & $1.010(0.863$ to $1 \cdot 183)$ & $1.023(0.819$ to $1 \cdot 280)$ & $1.126(0.927$ to 1.368$)$ & $0.996(0.853$ to 1.164$)$ \\
\hline Population & $1 \cdot 000^{\star \star}(1 \cdot 000$ to $1 \cdot 000)$ & $1 \cdot 000^{\star \star}(1.000$ to 1.000$)$ & $1.000(1.000$ to 1.000$)$ & $1.000 *(1.000$ to 1.000$)$ \\
\hline Area (square km) & $1.009^{\star \star}(1.000$ to $1 \cdot 017)$ & $1.016^{\star \star \star}(1.005$ to 1.028$)$ & $1.008(0.997$ to 1.019$)$ & $1.006(0.999$ to 1.014$)$ \\
\hline \multicolumn{5}{|l|}{ Mother's education } \\
\hline Primary & $1.243(0.881$ to $1 \cdot 755)$ & 1.544 (0.887 to $2 \cdot 686)$ & $1.229(0.816$ to $1 \cdot 850)$ & $1.282(0.914$ to 1.798$)$ \\
\hline Secondary & $1.522^{*}(0.972$ to 2.385$)$ & $2 \cdot 023^{\star \star}(1.030$ to 3.977$)$ & $1.516(0.875$ to $2 \cdot 626)$ & $1.551^{*}(0.998$ to 2.409$)$ \\
\hline Higher & $0.854(0.184$ to 3.968$)$ & $2 \cdot 422(0.482$ to $12 \cdot 185)$ & $0.968(0.120$ to $7 \cdot 833)$ & $0.850(0.183$ to 3.940$)$ \\
\hline Age of mother & $1.004(0.985$ to 1.023$)$ & $0.996(0.968$ to 1.025$)$ & $1.004(0.981$ to 1.027$)$ & $0.996(0.977$ to 1.015$)$ \\
\hline Age of child & $0.896^{\star *}(0.821$ to 0.979$)$ & $0.893^{*}(0.785$ to 1.016$)$ & $0.973(0.875$ to 1.082$)$ & $0.908^{\star \star}(0.833$ to 0.989$)$ \\
\hline $\begin{array}{l}\text { Distance from } \\
\text { nearest Health } \\
\text { Facility }\end{array}$ & $0.963(0.898$ to 1.032$)$ & $0.947(0.850$ to 1.055$)$ & $0.947(0.872$ to 1.030$)$ & $0.963(0.900$ to 1.031$)$ \\
\hline \multicolumn{5}{|l|}{ Wealth Index } \\
\hline Poorer & $1.307(0.891$ to 1.918$)$ & $1.076(0.619$ to 1.871$)$ & $0.955(0.616$ to 1.481$)$ & $1.283(0.885$ to 1.862$)$ \\
\hline Middle & $0.982(0.657$ to 1.467$)$ & $0.707(0.387$ to 1.293$)$ & $0.661^{*}(0.411$ to 1.063$)$ & $0.961(0.651$ to 1.418$)$ \\
\hline Richer & $1 \cdot 130(0.760$ to $1 \cdot 679)$ & $0.775(0.433$ to 1.386$)$ & $0.938(0.599$ to 1.471$)$ & $1.125(0.766$ to 1.653$)$ \\
\hline Richest & $1 \cdot 109(0.696$ to $1 \cdot 765)$ & $0.818(0.426$ to 1.571$)$ & $0.697(0.393$ to 1.235$)$ & $1.094(0.694$ to 1.724$)$ \\
\hline \multicolumn{5}{|c|}{ Geographic classification } \\
\hline Rural & $0.831(0.526$ to 1.312$)$ & $0.650(0.349$ to 1.211$)$ & $1 \cdot 157(0.648$ to $2 \cdot 064)$ & $0.940(0.599$ to 1.473$)$ \\
\hline $\begin{array}{l}\text { Malaria Ecology } \\
\text { Index }\end{array}$ & $2 \cdot 477^{\star \star \star}(1.504$ to 4.079$)$ & $2 \cdot 187^{\star \star}(1.067$ to $4 \cdot 485)$ & $1.995^{\star \star}(1.090$ to $3 \cdot 650)$ & $2.384^{\star \star \star}(1.462$ to 3.890$)$ \\
\hline Constant & $0.071^{* \star *}(0.021$ to 0.239$)$ & $0.019^{\star \star *}(0.003$ to 0.112$)$ & $0.052^{* * \star}(0.012$ to 0.231$)$ & $0.089^{* \star *}(0.027$ to 0.294$)$ \\
\hline
\end{tabular}

Reference group includes low malaria service readiness, low malaria diagnostic readiness, low malaria training readiness, no education, poorest and urban geographic location.

${ }^{\star * \star \star *} \mathrm{P}<0.01,{ }^{\star \star} \mathrm{P}<0.05,{ }^{\star} \mathrm{P}<0.1$.

$\mathrm{ACT}$, artemisinin-based combination therapy.

Further, these results appear to present conflicting evidence of the association between health aid and facility readiness such that aid appears inversely associated with facility readiness overall but positively associated with diagnostic capacity. This finding is interesting for several reasons. First, it indicates that health aid is translating to increased malaria diagnostic capacity. Early and accurate diagnosis of malaria is essential to lower mortality and stem the spread of disease. ${ }^{18}$ Diagnosis is also important, because not every fever contributes to malaria. Due to increasing concerns related to drug resistance, presumptive treatment is no longer recommended unless diagnostic tests are not accessible. ${ }^{18}$ Second, the finding that aid is translating into diagnostic capacity, but not overall facility readiness highlights a gap that could significantly impact the quality and accessibility of malaria diagnosis. Referring back to the Baron and Kenny model for steps 3 and 4, findings indicate that the coefficient for Pathway B was significant in two cases of overall malaria service readiness. This result is not surprising given the fact that $96 \%$ of health facilities in Malawi had full capacity to provide first-line treatment medicine. Individuals may have obtained effective treatment from facilities with a lower level of readiness. The results also suggest that both Pathways $\mathrm{C}$ and C' are similar and not statistically different from zero, suggesting there is neither a direct nor indirect (ie, mediated via facility readiness) association between health aid and malaria service utilisation. Therefore, mediation was not formally tested using a Sobel test. ${ }^{19}$ 


\section{Limitations and strengths}

Several limitations should be acknowledged when interpreting these results. First, it is not possible to directly link the MIS to the facility. Therefore, KDE was used to assign a density of malaria service readiness to each child. Although this method allows for the incorporation of distance decay as well as the possibility to assign an aggregate measure of readiness to each child, it does not reflect the true travel distance to the clinic. Second, since the KDE disperses the effect of a facility across space by facility characteristics; the malaria service readiness index was derived using an equal weighting additive approach for interpretability. One could argue that the items in the index have different clinical values and thus should be weighted differently. Since a weighted additive index has not been validated within the context of $\mathrm{KDE}$, there are still remaining assumptions that the dimensions do in fact carry equal weights. ${ }^{20}$ Third and related to the creation of the index, the malaria service readiness index ranked facilities as high, medium or low quality based on the distribution of the score. This was done in order to examine the differences among facilities while retaining a more intuitive understanding, Although it is a common practice in social science to dichotomise continuous variables, it may result in a loss of statistical power and result in an ability to detect significant differences. ${ }^{20-22}$ For this reason, I categorised the continuous variable into terciles. Discretisation in this way allows for the comparison of the highest and lowest groups and results in less efficiency lost. ${ }^{20}{ }^{23}$ Fourth, this work is based on the assumption that the readiness of the facility to provide malaria services influences the decision to use a facility. Parents may choose to use facilities for reasons unconnected to service readiness. ${ }^{13}$ Fifth, the SPA and MIS data were collected within 1 year of each other. The malaria service utilisation data preceded the service provision data used to determine service readiness. Therefore, this research assumes that the health facility service provision capacities did not change much between the two surveys. Although this follows DHS guidelines for linking SPA and MIS data, data on malaria service availability collected during the SPA survey could have changed after the MIS survey. ${ }^{24}$ Sixth, the MIS data describe the 2-week period of prevalence of fever among children under five. While a history of fever is often strongly associated with malaria parasitaemia in malaria-endemic countries, children who had a fever may not have had malaria. Seventh, I restrict the AidData dataset to health aid projects that could feasibly have local impacts on malaria services and utilisation. However, I cannot subset the data to only include malaria-specific funding. In addition, AidData does not assign financial amounts to individual project locations. Many researchers choose to divide the total aid amount equally across all activities. In order to test the sensitivity of my results to other definitions of aid, I repeated the analysis defining health aid in terms of the dollar amount across each region. The results of this analysis were qualitatively similar to the more rigorous definition of aid based on counts of health aid projects that were used in this study. Also, this research uses date of planned completion to subset the timing of aid distribution. However, from this dataset, it is not possible to know exactly how much of this aid was actually transferred to the areas in question. A long lag between aid and the period the surveys were conducted may attenuate the association between aid and the outcomes of interest. In addition, enough time may not have elapsed for changes to occur in facility readiness that influence utilisation. In order to test the sensitivity of my results to temporality and endogeneity, I examined aid in two bins of early and late aid. Results of this analysis are located in online supplementary appendix $\mathrm{G}$ and indicate that it is appropriate for future research to consider binning aid into early and late periods. Eighth, this analysis assumes that health aid mainly influences the supply side of malaria care. It is possible that aid is used to create demand for malaria care and subsequently increase service use. Although, in theory improved quality of care can stimulate demand for care, in a resource constrained country like Malawi, cost associated with seeking care are formidable and unless these are addressed, improving facility readiness may not stimulate service use..$^{25}$ Ninth, this analysis does not explicitly control for government and private investments in health that foreign donors do not fund, which could bias estimates by underestimating the aid amount. However, the Malawi government's contribution to health expenditure has been consistently below the $15 \%$ recommended in the Abuja declaration, estimated at $7.2 \%$ in $2011 .{ }^{26}$ Consequently, any bias from excluding government funded interventions should be minimal. Tenth, the cross-sectional nature of both the SPA and the MIS prevent more rigorous estimates of the influence of service readiness on service utilisation. Specifically, I cannot examine whether changes in health aid in a geographic region cause changes in malaria facility readiness or utilisation.

Despite these limitations, this study has several noteworthy strengths. First, this paper linked facility census data and nationally representative household data to provide a more robust assessment of whether and how health aid, facility readiness to provide malaria services and the use of malaria services relate. ${ }^{13}$ The use of KDE to join these data provides a methodological improvement in the way the service environment was constructed and linked to household survey data. Similar to Wang, but with a different empirical approach, my analysis incorporates distance decay to measure the effect of the malaria service environment instead of only looking at the closest facility, reducing the likelihood that individuals were misclassified to a specific facility. ${ }^{13}$ Second, this is the first study to my knowledge to combine subnational aid flows with individual-level and facility-level measures of malaria service availability and use. Third, the mediation approach was innovative in its attempt to provide evidence on potential mechanisms by which health aid and malaria utilisation relate and identifies a need to strengthen or improve measurement of programme components.

\section{CONCLUSION}

The overall evidence presented suggests that utilisation of malaria services among children under five living in 
Malawi is low. Further, almost all types of health facilities had diagnostic capacity as well as ACT available, but there was limited capacity in terms of training and protocols. Using a mediation approach popularised by Baron and Kenney and rooted by the Andersen-Aday conceptual framework for health services utilisation, this study found little support for the overarching hypothesis that health aid boosts a facility's readiness to provide malaria services and that facility readiness increases utilisation of malaria services. Although the results did not find a significant association between health aid and service utilisation (Research Question 1), a positive association was found between health aid and certain domains of facility readiness, namely malaria diagnostic capacity (Research Question 2). Accurate and early diagnosis is one of the key strategies to control and prevent malaria and confirmation that health aid influences readiness to provide early detection is notable. ${ }^{18}$ The finding that health aid was positively associated with diagnostic capacity, but not positively associated with overall facility readiness, indicates a clear gap for funders to consider when developing aid allocation strategies. In Malawi, health aid does contribute to the availability of diagnostic tools, but without an overall facility readiness to implement these diagnostic tools, the quality and accessibility of malaria diagnosis is limited. Future funding policies should consider a mechanism for comprehensive and consistent training in the use of diagnostic tools. Future research should collect and leverage aid data that distinguishes among the type of health aid (eg, malaria-specific aid) as well as timing the aid to better identify the processes by which donor investments strengthen health systems and, ultimately, improve health. More evidence is needed to increase the understanding of the mechanisms that determine service utilisation in developing countries and in order to identify which critical programme components of healthcare systems require donor investment.

\begin{abstract}
Acknowledgements Valuable assistance as well as helpful and timely comments were provided by Ariel BenYishay, Andrew Barnes, Mead Over, April Kimmel, David Wheeler, HP, and Kate Epstein. I also thank the anonymous reviewers for their
\end{abstract} careful reading of this research and for their insightful comments and suggestions.

Contributors CBD conceived and designed the study, acquired and analysed the data as well as drafted the manuscript for submission.

Funding The authors have not declared a specific grant for this research from any funding agency in the public, commercial or not-for-profit sectors.

Competing interests None declared.

Patient consent Not required.

Ethics approval This research uses secondary data which is deidentified and therefore IRB approval is not required.

Provenance and peer review Not commissioned; externally peer reviewed.

Data sharing statement Data can be accessed by emailing the corresponding author Carrie Dolan cbdolan@wm.edu.

Open access This is an open access article distributed in accordance with the Creative Commons Attribution Non Commercial (CC BY-NC 4.0) license, which permits others to distribute, remix, adapt, build upon this work non-commercially, and license their derivative works on different terms, provided the original work is properly cited, appropriate credit is given, any changes made indicated, and the use is non-commercial. See: http://creativecommons.org/licenses/by-nc/4.0/

\section{REFERENCES}

1. World Health Organization, 2017. Cost effectiveness and strategic planning (WHO-CHOICE). Available from: http://www.who.int/choice/ globalpricetags/en/

2. Wroe D. Donors, dependency, and political crisis in Malawi. Afr Aff 2012;111:135-44.

3. The United Nations Children's Fund, (UNICEF). Health budget brief 2016/17. Malawi, 2017.

4. Dionne KY. Donor dependence, donor withdrawal: implications of Malawi's cashgate scandal. AidData Beta: Open Data for International Development, 2014

5. Okiro EA, Kazembe LN, Kabaria CW, et al. Childhood malaria admission rates to four hospitals in Malawi between 2000 and 2010. PLoS One 2013;8:e62214.

6. Andersen R, Newman JF. Societal and individual determinants of medical care utilization in the United States. Milbank Mem Fund Q Health Soc 1973;51:95-124.

7. Baron RM, Kenny DA. The moderator-mediator variable distinction in social psychological research: conceptual, strategic, and statistical considerations. J Pers Soc Psychol 1986;51:1173-82.

8. MEASURE Evaluation, MEASURE DHS, President's Malaria Initiative, Roll Back Malaria Partnership, UNICEF, World Health Organization. Household suvery indicators for malaria control, 2013.

9. Marty R, Dolan CB, Leu M, et al. Taking the health aid debate to the subnational level: the impact and allocation of foreign health aid in Malawi. BMJ Glob Health 2017;2:e000129.

10. Grépin KA, Fan VY, Shen GC, et al. China's role as a global health donor in Africa: what can we learn from studying under reported resource flows? Global Health 2014;10:84

11. National Malaria Control Programme (NMCP) [Malawi] and ICF International. Malaria Indicator Survey MIS. Malawi, 2012.

12. Wang W, Wang S, Pullum T. How family planning supply and the service environment affect contraceptive use: findings from four east African countries. DHS analytical studies No.26, 2012.

13. Wang W, Winner M, Burgert $C$. Influence of service readiness on use of facility delivery care: a study linking health facility data and population data in haiti. DHS working papers No. 114, 2014.

14. Loeys T, Moerkerke B, Vansteelandt S. A cautionary note on the power of the test for the indirect effect in mediation analysis. Front Psychol 2014;5:1549.

15. O'Rourke HP, MacKinnon DP. When the test of mediation is more powerful than the test of the total effect. Behav Res Methods 2015;47:424-42.

16. Rucker DD, Preacher KJ, Tormala ZL, et al. Mediationanalysis in social psychology: current practices and new recommendations. Soc Personal Psychol Compass 2011;5:359-71.

17. Mackinnon DP, Dwyer JH. Estimating mediated effects in prevention studies. Eval Rev 1993:17:144-58.

18. World Health Organization. World malaria report, 2013.

19. Sobel ME. Asymptotic confidence intervals for indirect effects in structural equation models. Sociol Methodol 1982;13:290-312.

20. Mallick L, Wang W, Temsah G. A comparison of summary measures of quality of service and quality of care for family planning in Haiti, Malawi, and Tanzania 2017.

21. Aiken LS, West SG, Reno RR. Multiple regression: testing and interpreting interactions. Sage, 1991.

22. MacCallum RC, Zhang S, Preacher KJ, et al. On the practice of dichotomization of quantitative variables. Psychol Methods 2002;7:19-40.

23. Gelman A, Park DK. Splitting a predictor at the upper quarter or third and the lower quarter or third. Am Stat 2009;63:1-8.

24. Burgert-Brucker CR, Prosnitz D. Linking DHS household and SPA facility surveys: data considerations and geospatial methods. ICF International, 2014

25. Ochalek J, Revill P, Manthalu G, et al. Supporting the development of a health benefits package in Malawi. BMJ Glob Health 2018;3:e000607.

26. Borghi J, Munthali S, Million LB, et al. Health financing at district level in Malawi: an analysis of the distribution of funds at two points in time. Health Policy Plan 2018;33:59-69. 